\title{
13
}

\section{Principles of Assessment and Treatment}

\begin{abstract}
In this chapter we outline the key principles that are built into the structure of our Mind-Body Program and into the interventions we undertake with any particular child. The principles are especially useful in the following circumstances: in assessing a referral for treating functional somatic symptoms; in setting up the multidisciplinary team around a given patient; in explaining to the child and family the overall goals and structure of the treatment approach to be used; in determining the details of the therapeutic interventions to be used; in discussing and initiating those particular interventions with the child and family; in periodically assessing the success or failure of ongoing therapeutic interventions; and when confronting situations in which the therapy feels stuck and the treatment interventions that should be working are not working. In addition, the principles make key processes explicit and can be used as touchstones for teaching and for learning. As touchstones, the principles can be used to identify dynamics both within the family and between the clinician and the family, and to help to open space for reflection about
\end{abstract}

Electronic supplementary material The online version of this chapter (https://doi.org/10.1007/978-3-030-46184-3_13) contains supplementary material, which is available to authorized users.

(C) The Author(s) 2020

K. Kozlowska et al., Functional Somatic Symptoms in Children and Adolescents, Palgrave Texts in Counselling and Psychotherapy, https://doi.org/10.1007/978-3-030-46184-3_13 
different aspects of the therapeutic process. Specific interventions, organized by system levels, are discussed in Chapters 14-16.

The treatment of children (including adolescents) with functional somatic symptoms and their families ranges from straightforward to fraught with difficulty, and anywhere in between. To understand and manage this complexity, the mental health clinician — or more commonly, the multidisciplinary clinical team (from now on, simply the clinician) — needs to keep in mind some key principles. The principles help make key processes explicit-those that take place in the paediatric setting, the mental health setting, and between the clinician and family to support a path to health and well-being (versus illness and dis-ease). The principles also help us, the clinician, to effectively conceptualize and reflect about processes and patterns of communication between the family and medical system, within the family itself, and between the clinician and family. Keeping these principles in mind is helpful because their implementation increases the likelihood that the child will return to a state of health and well-being. We discuss these principles one by one, clustered under headings that approximate the temporal order of the therapeutic process. The reader will notice, however, that some of the principles are overlapping and interdependent, and that in clinical practice they are applied in tandem. Notwithstanding, we provide all the principles here as a sort of map that clinicians can use to guide the treatment process or that they can consult when they feel lost or when the therapeutic process is not going well.

\section{Setting Things Up in the Broader Health Care System: Principles Concerning the Paediatrician and the Medical Assessment Process}

\section{Principle 1: Completion of a Thorough Medical Assessment}

This principle pertains to processes in the health care system that take place before the mental health clinician accepts a referral. As we have 
noted throughout the book, best practice in the field of functional somatic symptoms requires that a thorough medical assessment-by a paediatrician or family doctor - be completed prior to referral to a clinician for treatment. As part of the medical assessment, the paediatrician needs to have ruled out an organic cause for the symptoms, identified any concomitant medical factors that are part of the child's presentation, and identified the pattern of symptoms and signs that enabled the paediatrician to provide the child and family with a positive diagnosis of a functional disorder (for positive diagnosis, see Chapter 2 and Online Supplement 2.1). In providing a positive diagnosis, the paediatrician needs to tell the child and family explicitly that the child's pattern of symptoms and signs has a name-for example, functional abdominal pain or functional neurological disorder-and that specific treatment is required. Via this medical process that precedes the referral to the mental health clinician, the paediatrician contributes to the creation of a secure base from which the child, family, and clinician can feel safe enough to explore the various factors that contributed to the child's presentation. Without this secure base, neither the family nor the mental health clinician can engage in the therapeutic process, because they will remain concerned that an organic condition may have been missed.

\section{Principle 2: Early Referral for Mind-Body Treatment by the Paediatrician Leads to Good Outcomes and a Return to Health}

The literature suggests that children with functional somatic symptoms have better outcomes than adult patients and that their functional symptoms are more likely to remit. Our own clinical experience is that when children are referred early to established programs-or treatment teams or settings - specifically developed for, or designed to include, children with functional somatic symptoms, they achieve much better clinical outcomes than children whose referrals for treatment have, for one reason or another, been significantly delayed. From the perspective of the stress-system model, it seems likely that early referral and treatment facilitate settling of the child's stress system to a more regulated 
state, preempt the decline into chronicity, and prevent the establishment of illness-reinforcing patterns within the family, school, and medical systems (see Chapter 2). Clinicians working in hospital settings and those who have ongoing relationships with paediatricians should advocate for, and take every opportunity to advocate for, early referral of functional disorders to appropriate mental health clinicians (Tot-Strate et al. 2016; Garralda 2016).

\section{Setting Things Up in Psychological Medicine or the Mental Health System: Principles Concerning the Mental Health Clinician and Mental Health Setting}

\section{Principle 3: Maintaining a Systems Perspective}

Thinking systemically about the problem of functional somatic symptoms involves 'thinking in terms of relationships, patterns, and contexts' (Capra and Luisi 2014, p. xii). The activation and dysregulation of the child's stress system, along with the emergence of functional somatic symptoms, occur in the context of interactions between the child (her body, brain, and mind) and the world outside (her experience of, and interactions within, the home, school, and broader sociocultural contexts) - interactions that Hans Selye referred to as the stress of life (Selye 1956) (see Online Supplement 1.2). In this context, the clinician needs to be able to hold in mind different system levels and the relationships between them. Rather than focusing on the symptoms(s) and treating the symptoms per se, with each new patient the clinician is challenged to identify and address the specific areas of dysfunction-on the body, individual, family, school, and social-system levels-that contribute to stress-system activation and dysregulation. In this way, the treatment may potentially involve interventions that engage and target all these system levels. While each intervention on its own will effect only a small change within the stress system, multiple targeted interventions across system levels will achieve considerable change. In addition, because components 
within the stress system are interrelated and interconnected, change in one component can lead to changes in other components-what are sometimes referred to as ripple effects (Guite et al. 2012).

See Online Supplement 13.1 for the many different terms have been used to describe this broader (systemic) way of working with children and their families and for published case studies using the systems approach to treatment.

\section{Principle 4: The Goal of Treatment Is to Re-establish Regulation, Harmony, and Coherence Within the Child's Stress System}

Principle 4 follows on directly from Principle 3. Clinicians who work with functional somatic symptoms using a systemic perspective see the overarching goal of the therapeutic intervention as helping the child to re-establish regulation, harmony, and coherence within her stress system, enabling the functional somatic symptoms to resolve (see Fig. 13.1). Put simply, the clinicians are treating a system-the child's stress system and all the factors that activate the stress system — and not the symptoms per

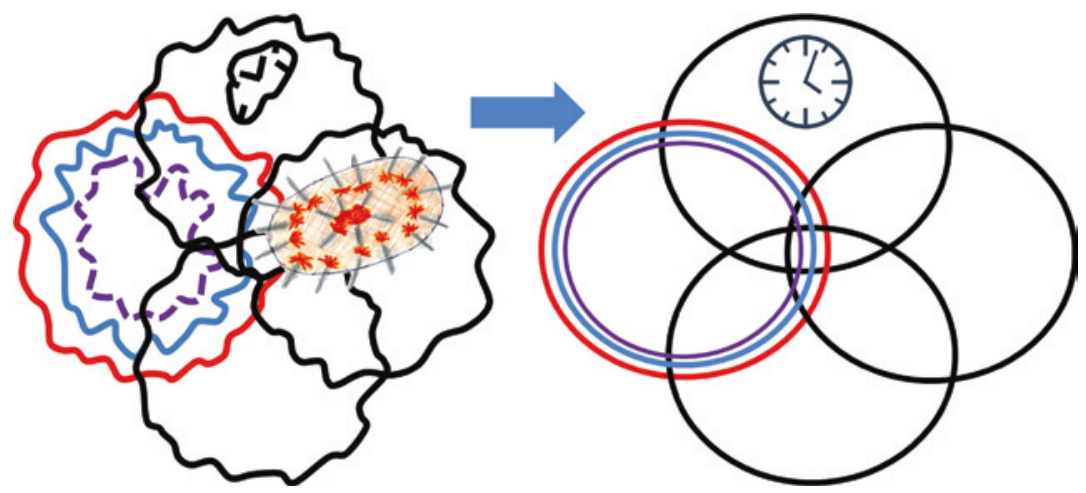

Fig. 13.1 Treatment and healing depicted using the circles metaphor of the stress system. With appropriate interventions, a dysregulated stress system that supports and generates functional somatic symptoms shifts to a regulated stress system that supports health and well-being (๑ Kasia Kozlowska 2017) 
se. If all goes well, the child will develop an increased capacity for managing stress and distress; nurturing relationships will have been strengthened; the family will have made changes that support health and the child's well-being; and the stress in the family, school, and broader social environment will have been resolved. The overall result will be to build the child's resilience and to buffer her from future stress.

\section{Principle 5: Establishing a Multidisciplinary Team to Work with the Child and Family}

A multidisciplinary team is formed to enable the implementation of a therapeutic intervention on multiple system levels. Because the interventions at different system levels involve different skill sets, the treatment team for the child with functional somatic symptoms typically includes a diverse range of professionals who work together in a coordinated way. In the inpatient setting where the first author $(\mathrm{KK})$ works, the multidisciplinary team always consists of a psychiatrist, a clinical psychologist, a physiotherapist, a paediatric resident on rotation, nursing staff from the ward, and school staff from the hospital school. When the need arises, an occupational therapist, neurologist, or paediatrician will also join the team.

\section{Principle 6: Begin the Treatment Process with Interventions That Target the Body, and Move On to Interventions That Target the Mind and the Family System}

Because the child presents with symptoms that pertain to the body, both the child and family usually find it easier to become engaged in the treatment-which will eventually reach multiple systems-when the clinician begins with an intervention targeting the body system. For example, in the first author's program, the interventions implemented first include stabilization of sleep, mind-body interventions designed to ameliorate the child's physical well-being, physiotherapy to address functional neurological symptoms or to build resilience, and attendance 
at the hospital school. Once the child's symptoms have been stabilized to some degree-and everyone can see that the child's physical well-being is on the mend-both the child and family find it easier to move on to interventions at the mind and family system levels (see, for example, the vignette of Paula in Chapter 3). Using family systems parlance, this is a strategic systemic approach in which the clinician begins with interventions on a system level that is more intuitive and acceptable for the child and family and then moves to system levels that are more challenging.

\section{Principle 7: The Goal of Treatment Is a Return to Health/Well-Being and Normal Activities}

For clinicians working with children, the goal of treatment should be a full return to health (for outcome data see Online Supplement 2.1). This principle is stated explicitly because the situation is different in adult practice, in which chronic functional presentations are more common and in which the probability that the symptoms will fully remit is lower. Clinicians working with adults often focus on helping the patient accept the illness; they support the patient in engaging in treatment to modify the symptoms to some degree; and they support the patient to accept that she or he may have to continue living with some degree of disability. By contrast, clinicians working with children should focus on getting the child well. In other words, in working with children, the clinician should voice the expectation that the treatment will enable the child to shift her stress system from defensive to restorative mode and that her functional somatic symptoms will resolve. During the treatment process, the child will begin the process of resuming her normal activities and should expect, with time, to return to a state of health and well-being. Of course, if there are comorbid chronic health concerns or unresolvable predicaments that cannot be modified, this expectation about returning to full health may itself need to be modified. In such scenarios the working goal may be a return to the child's previous level of function and activities, or alternatively, to her best possible level of function. 


\section{Principle 8: Understanding and Managing Attention Plays a Central Role in Achieving and Maintaining Progress Across System Levels and Across Treatment Interventions}

Attention to functional somatic symptoms amplifies such symptoms, and attention away from the symptoms diminishes them (see Chapter 12). In this context, careful management of attention is a key element across all treatment components during the treatment intervention. In the first author's Mind-Body Program, the change in focus of attention is reflected in the very structure of the program (Kozlowska et al. 2012). Parents are asked to give the child space by absenting themselves from the hospital during the daytime hours that the child is engaged in the program. In this way, parents no longer attend to the child's symptoms day in and day out. Instead, the child is helped to attend to normal daily activities such as attendance at school and to activities that take place in physiotherapy and psychotherapy sessions. Likewise, the transition from assessment to treatment involves a shift in the focus of attention: the clinician shifts the focus of attention away from the symptoms to the mind-body strategies that the child is learning to use in managing the symptoms and also to the goals pertaining to functional gains (e.g., getting up in the morning, walking for longer distances, and going to school). For this attentional component of the intervention to work, parents must likewise learn (as part of the family-level intervention) to shift their attention away from the symptoms, to focus on supporting the child in implementing her new mind-body strategies, and to attend to the factors within the family system contributing to the child's symptoms. Likewise, the management of attention is a key component of physiotherapy (Gray et al. 2020) (see vignette of Jai in Chapter 15).

\section{Principle 9: Clinicians Need to Remain Adaptable, Flexible, Creative, and Open to New Information}

Openness and adaptability to new information are important for professionals working with functional somatic symptoms; the current 
knowledge base is limited and ever evolving, and all current models and theories reflect approximate truth (Capra and Luisi 2014).

\section{Meeting the Family and the Engagement Process: Listening to the Story, Co-constructing a Formulation, Treatment Planning, Setting Expectations, and Agreeing to a Treatment Contract}

\section{Principle 10: Establishing a Therapeutic Relationship with the Multidisciplinary Team}

Establishing a therapeutic relationship with the child and family is fundamental. A solid relationship enables the child's parents to give their unqualified approval (consent) to the treatment intervention (thereby giving the child explicit permission to be open and honest in her individual work); it bolsters the parents' effort to support the child throughout the treatment process (especially when the going gets tough); and it enables the clinician to address parental and family behaviours and factors that contribute to the child's illness. In building a therapeutic relationship, the clinician continues the process of solidifying the secure base-as initially set in motion by the paediatrician (see Chapter 2)that is a prerequisite for a successful therapy intervention.

When parents and the child with functional somatic symptoms connect emotionally with the clinicians who make up the treatment team, anxiety within the family system settles to some degree. This settling of anxiety facilitates the parents' regulation of their own body statesthe degree to which their stress systems are activated-and increases the probability that the parents will be able to support their child and efforts to settle the child's stress system.

A good therapeutic relationship also promotes positive and playful interactions between the child, family, and treatment team. Positive interactions work to counteract the defensive body state in which the child finds herself. In this way, positive interactions contribute to the 
neurophysiological conditions that Stephen Porges refers to as the neural platform - the range of body states underpinning better regulation and associated with health and well-being (Porges and Furman 2011).

\section{Principle 11: Using a Working Formulation to Hold in Mind Information from Different System Levels}

The formulation - the creative synthesis of a clinical case-draws on elements from all system levels, including the child's stress system, the child's mind, interactions between the parent and child, the contribution of the family, and experiences in the school context (Ross 2000; Winters et al. 2007). As we saw in Chapter 3, the formulation is co-constructed: it is worked out collaboratively in the conversation with the child and family during the assessment process. In this way, the formulation is a story that integrates, in chronological order, all the factors that contributed-and that currently contribute-to the child's functional illness and her clinical presentation. It is unique and specific to a child and her family. The formulation is always a working formulation; that is, it is modified during the treatment process as the clinician learns more about the child and family, and as trust is established and the family becomes more willing to reveal more about themselves and about their understanding of the factors that contributed to the child's presentation. In this way, as with all working hypotheses, the formulation is updated and changed - by the clinician and family members-as new information comes to light.

\section{Principle 12: Use the Formulation to Guide the Choice of Treatment}

In collaboration with the child and family, the clinician uses the formulation to guide the choice of treatment strategies, techniques, and interventions to promote change on the body, brain, mind, behavioural, family, and school and social-system levels. The process of implementing the multifaceted intervention involves the specific strategies, techniques, 
and interventions that are implemented - by the child, family, school, and clinical team-on each of those separate system levels. Each intervention is chosen because it targets a problem area identified in the co-constructed formulation (see the case of Paula in Chapter 3), because it represents a nodal point for intervention (see below), or because it is seen as essential to health and well-being, both now and in the long term. See Online Supplement 13.1 for published case examples as to how the formulation is used to guide the choice of treatment.

\section{Principle 13: Identify Nodal Points for Intervention and Achieving Change}

The notion of nodal points of change is helpful when the treatment involves interventions that engage and target multiple system levels. Nodal points are interventions that result in a greater degree of change than others or are so fundamental that without them, the desired progress will not be achieved. Understanding the nodal points for any particular patient not only facilitates the choice of treatment but helps the clinician (and family) decide the temporal order in which the interventions will be implemented. For example, interventions that stabilize sleep-the circadian clock-are powerful because the circadian clock regulates all the components of the stress system, and regulation of the circadian clock is likely to increase coherence of the stress system as a whole. Good sleep also increases the child's capacity to regulate more generally and to engage in positive interactions with others. Another example pertains to maltreatment. A child who continues to be exposed to ongoing maltreatment-for example, cyberbullying that involves texts telling her to kill herself or telling her that she is worthless and should die-will not be able to down-regulate her stress system. Establishing safety is fundamental; other interventions cannot be expected to work until safety had been established. Yet another example pertains to a good diet. Maintaining the health of the gut microbiota is essential to the health and well-being of the individual because the microbiota lives in symbiosis with humans and works together with the human body to maintain life processes (see Chapter 10). 


\section{Principle 14: Engage Hope and Positive Expectations at Assessment and Throughout the Treatment Process}

The expectations and hopes that the clinician voices to the child and family_about the positive expectations for a successful outcome, about how the therapy achieves its results and how it will help the child to build her skills in managing her symptoms, and about the family's crucial role in supporting the child-are important because they function to activate the placebo effect in both the child and the family. Positive expectations and the placebo effect increase the likelihood that each component of the treatment program will have its maximal benefit (Benedetti 2011a, b, 2013). Because engagement of positive expectations is both a principle and an intervention, it is further discussed in Chapter 14.

\section{Principle 15: Agree to a Treatment Contract Before Treatment Begins}

The contract outlines expectations, roles, and responsibilities for the child, parents, and family, as well as for the treating team. It also defines expectations about the treatment intervention itself, the issues that will be addressed in the current intervention, and the issues that may need longer-term work with a different clinician or clinical team. The contract establishes a common understanding of, and agreement concerning, the key ingredients needed for change. It also minimizes the likelihood that the child and family will encounter unpleasant surprises about the treatment process - which is especially important since the program is challenging, demanding, and confronting for both the child and the family. For example, it is helpful for parents to know upfront that the child will be attending her physiotherapy sessions, her psychotherapy sessions, and the hospital school on her own, without the presence of a parent.

If the family does not want to agree to the contract, if parents want to try providing treatment at home, or even if they change their mind about the treatment intervention midway, then the family can decline the intervention or withdraw the child from the program, and set up elsewhere (if at all) their own preferred way of working. The family 
always has the option of re-engaging with the program-with its particular structure and expectations - at some future time.

\section{The Treatment Process: Effecting Change, Returning to Normal Function, and the Longer Journey to Maintain Health and Well-Being}

\section{Principle 16: The Treatment Is the First Step in a Longer-Term Process of Maintaining Health and Well-Being}

Early during the treatment process for functional somatic symptoms, it can be useful to highlight that the treatment intervention offered by the clinical team is just the first step in a longer journey of maintaining health and well-being. That journey may involve ongoing therapeutic work on different system levels_-individual, family, school, and so on. Common elements that need integration into the home routine include regular sleep and exercise, healthy eating, ongoing therapy for anxiety or depression, acquisition and maintenance of stress-management skills, improvement of family communication skills (e.g., via a family intervention), and system interventions that ensure resolution of ongoing stress in the family, school, and peer contexts. Ongoing attention to all these risk factors will build resilience, decrease the likelihood of relapse, and increase the probability of long-term health and well-being.

\section{Principle 17: For the Treatment Intervention to Work, Everyone-Child, Family, and Multidisciplinary Team-Needs to Be Pushing in the Same Direction}

All members of the multidisciplinary team, all members within the family unit (including, insofar as possible, grandparents and extended family), and all members of the school team need to share the formulation and support the treatment process. This same principle applies to 
interventions that take place within a health facility and those that take place in the community setting. In either setting, regular meetings and a written, updated treatment plan can be important tools in supporting a common understanding and in helping everyone involved in the child's care to push in the same direction. For example, an intervention will not work if the child's parents are supporting the child to complete her exercise program and her grandparents are simultaneously telling her that she should rest because her legs do not work normally and because she has pain and experiences fatigue.

\section{Principle 18: Normal Activities Are Both the Treatment and the Goal}

Many families hold the idea that the treatment process will first involve resolution of the child's symptoms and, second, resumption of normal activities. When treating functional somatic symptoms, resumption of normal activities - sometimes in a graded fashion - is part of the treatment itself, and this aspect of treatment begins alongside and at the same time as other interventions. As part of the conversation with the child and family, the clinician may also communicate that some symptoms-for example, pain, nausea, and fatigue-will typically resolve only after the child has been engaging in her normal activities for some period of time.

\section{Principle 19: Promote Predictability, Control, and Mastery for the Child}

The goals of predictability, control, and mastery (from the child's perspective) should guide the choice of treatment interventions alongside the specific areas of dysfunction identified by the clinician. Interventions that contribute to these goals promote health and well-being because they function to settle the stress system as well as to minimize activation of psychological processes on the mind system level that appraise the treatment intervention as threatening (see Chapter 12). 


\section{Principle 20: Self-Management Is Key to the Child Maintaining Stable, Long-Term Regulation of the Stress System}

A crucial factor in recovery is the child's willingness-with the support of her parents - to work on mind-body strategies that enable her to down-regulate the stress system and, more broadly, to improve her capacity for neurophysiological regulation. Fundamentally, the child is the only person who can, moment to moment-on waking up, at school, on coming home, and so on-implement the mind-body strategies that will help her to settle her stress system and to shift it from defensive to restorative mode. Without the child's cooperation and engagement, no one can accomplish this change in body state. Consequently, there is much to gain in giving the child the choice as to which mind-body strategies she enjoys and therefore wants to prioritize in her treatment plan. Creativity, along with respect for the child's choices, results in a treatment program that is shaped —and owned-by the child. And when these efforts to regulate and to manage her own body meet with success, it promotes a sense of control, mastery, and motivation, which are essential for long-term progress and stability (see Principle 19). This approach is consistent with data from the pain literature suggesting that increases in children's readiness to self-manage pain are associated with decreased functional disability, depressive symptoms, and fear of pain, and with increased use of adaptive coping strategies (Walker et al. 2006).

In this chapter we have highlighted the overarching considerations that, under the stress-system model, structure and guide the treatment of children with functional somatic symptoms. We have also presented, in particular, some of the key principles that we, as clinicians, find helpful when working with these children. When colleagues ask for help with specific cases, we often find it useful to bring these principles to bear on the clinical situation. And when the going gets tough in our own clinical work, we often return to these principles to potentially realign 
our efforts and to check that we have not omitted some key element that could help the child and family back toward a path of health and well-being. In this way the principles function as a map that can be used to guide the treatment process or that provides guidance when the going gets tough.

\section{References}

Benedetti, F. (2011a). Meeting the Therapist: A Look into Trust, Hope, Empathy, and Compassion Mechanisms. In The Patient's Brain. Oxford: Oxford University Press.

Benedetti, F. (2011b). Receiving the Therapy: The Activation of Expectation and Placebo Mechanisms. In The Patient's Brain. Oxford: Oxford University Press. Benedetti, F. (2013). Placebo and the New Physiology of the Doctor-Patient Relationship. Physiological Reviews, 93, 1207-1246.

Capra, F., \& Luisi, P. L. (2014). The Systems View of Life: A Unifying Vision. Cambridge: Cambridge University Press.

Garralda, M. E. (2016). Hospital Management of Paediatric Functional Somatic Symptoms. Acta Paediatrica, 105, 452-453.

Gray, N., Savage, B., Scher, S., \& Kozlowska, K. (2020). Psychologically Informed Physiotherapy for Children and Adolescents with Functional Neurological Symptoms: The Wellness Approach. Journal of Neuropsychiatry and Clinical Neurosciences.

Guite, J. W., Logan, D. E., Ely, E. A., \& Weisman, S. J. (2012). The Ripple Effect: Systems-Level Interventions to Ameliorate Pediatric Pain. Pain Management, 2, 593-601.

Kozlowska, K., English, M., Savage, B., \& Chudleigh, C. (2012). Multimodal Rehabilitation: A Mind-Body, Family-Based Intervention for Children and Adolescents Impaired by Medically Unexplained Symptoms. Part 1: The Program. American Journal of Family Therapy, 40, 399-419.

Porges, S. W., \& Furman, S. A. (2011). The Early Development of the Autonomic Nervous System Provides a Neural Platform for Social Behavior: A Polyvagal Perspective. Infant and Child Development, 20, 106-118.

Ross, D. E. (2000). A Method for Developing a Biopsychosocial Formulation. Journal of Child and Family Studies, 1, 106.

Selye, H. (1956). The Stress of Life. New York: McGraw-Hill. 
Tot-Strate, S., Dehlholm-Lambertsen, G., Lassen, K., \& Rask, C. U. (2016). Clinical Features of Functional Somatic Symptoms in Children and Referral Patterns to Child and Adolescent Mental Health Services. Acta Paediatrica, 105, 514-521.

Walker, L. S., Williams, S. E., Smith, C. A., Garber, J., Van Slyke, D. A., \& Lipani, T. A. (2006). Parent Attention Versus Distraction: Impact on Symptom Complaints by Children With and Without Chronic Functional Abdominal Pain. Pain, 122, 43-52.

Winters, N. C., Hanson, G., \& Stoyanova, V. (2007). The Case Formulation in Child and Adolescent Psychiatry. Child and Adolescent Psychiatric Clinics of North America, 16(111-132), ix.

Open Access This chapter is licensed under the terms of the Creative Commons Attribution-NonCommercial-NoDerivatives 4.0 International License (http://creativecommons.org/licenses/by-nc-nd/4.0/), which permits any noncommercial use, sharing, distribution and reproduction in any medium or format, as long as you give appropriate credit to the original author(s) and the source, provide a link to the Creative Commons license and indicate if you modified the licensed material. You do not have permission under this license to share adapted material derived from this chapter or parts of it.

The images or other third party material in this chapter are included in the chapter's Creative Commons license, unless indicated otherwise in a credit line to the material. If material is not included in the chapter's Creative Commons license and your intended use is not permitted by statutory regulation or exceeds the permitted use, you will need to obtain permission directly from the copyright holder.

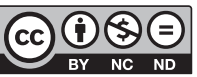

